\title{
Continuity, localization, and cosmology in warped geometry
}

\author{
Gregory Gabadadze, Daniel Older@, and David Pirtskhalava \\ Center for Cosmology and Particle Physics, Department of Physics New York University, \\ New York, New York 10003, USA
}

(Received 3 August 2021; accepted 23 September 2021; published 12 November 2021)

\begin{abstract}
This is the first of two papers studying localization of massive bulk fields on a bane in 5D anti-de Sitter spacetime, and some of their cosmological consequences. Here we focus on a massive 5D scalar, which is known to lack a localized mode, and discuss how a seeming discontinuity between this theory and the massless theory-known to support a localized zero mode-is resolved thanks to peculiar analytic properties of the massive two-point amplitude. Furthermore, we propose a boundary term that leads to the emergence of a massless localized mode in the massive theory. Last but not least, we consider the case when the brane world volume is de Sitter spacetime, and prove the existence of a localized massive mode. We discuss how these results, taken collectively, can be used to describe the accelerated expansion due to the massive 5D scalar field in an early, or in a late-time universe.
\end{abstract}

DOI: 10.1103/PhysRevD.104.104031

\section{INTRODUCTION AND SUMMARY}

Randall and Sundrum (RS) [1] have shown that a 5D massless graviton yields a localized massless $4 \mathrm{D}$ graviton in 5D AdS spacetime with a $Z_{2}$ symmetric brane; the rest of the Kaluza-Klein(KK) modes form a gapless continuum, with the wave functions for the lighter KK modes suppressed on the brane, leading to approximate recovery of 4D physics at low energies. ${ }^{1}$ The RS discovery has a numerous physical and theoretical consequences, which are well known.

It turns out that massless 5D scalar also yields a localized massless 4D mode on the brane worldvolume [4], with the gapless KK continuum and the KK wave functions identical to those of massless 5D graviton. However, massless vectors and spinors do not produce localized massless modes in the minimal setup.

Our goal is to consider whether massive spin- 0 , spin- 1 , and spin-2 fields can be localized or quasilocalized on a brane in $\mathrm{AdS}_{5}$. This is the first of the two papers on this topic; here we focus on a 5D massive scalar field to

\footnotetext{
${ }^{1}$ Note that $\mathrm{AdS}_{5}$ refers to the universal cover of $5 \mathrm{D}$ AdS spacetime [2]. There exists a stable solitonic domain wall solution with 4D Poincare invariant worldvolume, and $Z_{2}$ symmetry in the direction transverse to the worldvolume [3]. This solution, in a certain approximation, can be thought of as infinitely thin brane with all its fluctuations becoming negligible for a low energy brane observer.

Published by the American Physical Society under the terms of the Creative Commons Attribution 4.0 International license. Further distribution of this work must maintain attribution to the author(s) and the published article's title, journal citation, and DOI. Funded by SCOAP ${ }^{3}$.
}

emphasize some of the subtleties which are independent of the spin, but also to delineate cosmological scenarios specific to such a massive 5D scalar.

The question of localization of a 5D massive field was first studied in [5], where it was found that there is no localized mode irrespective how small the 5D mass is. Yet, there is a resonant mode, that can mimic physics of the zero mode in a certain approximation [5].

Seemingly, there is discontinuity between the massless and massive theory - the former has a massless 4D mode in its spectrum while the latter does not, irrespective how small the 5D scalar mass is. Indeed, this discontinuity is real as long as the spectra of the modes are concernedthe bulk mass makes the would-be localized mode nonnormalizable, for any nonzero value of the mass. However, we will show that there is no discontinuity in the two-point amplitude of the theory. This involves careful consideration of the analytic properties of this amplitude, as it is done in Sec. II.

In Sec. III we show how one can modify the brane world volume theory by adding a simple $4 \mathrm{D}$ term for the scalar to reinstate the massless $4 \mathrm{D}$ localized mode for a massive $5 \mathrm{D}$ bulk scalar field. The new term is a "tachyonic" 4D world volume mass for the scalar, which depends on the bulk mass. We should note, however, that the $4 \mathrm{D}$ term is overwhelmed by the positive 5D mass term and nowhere one encounters any tachyonic instabilities in the theory. The new term creates an additional "attraction" in a potential for the spectral problem for the KK modes, and this is enough to reinstate the massless mode.

In Sec. IV we consider 5D massive scalar in the background geometry with 4D de Sitter world volume. We prove the existence of a localized massive mode in this case 
and a KK continuum starting above the gap determined by the curvature of the 4D de Sitter spacetime. We then outline how such a geometry can be an approximation to the one on which 4D curvature is provided by a slowly rolling 5D massive scalar field. We point out differences of such a scheme from the one with 4D massive scalar slowly rolling and providing inflation or late time acceleration (quintessence).

\section{MASSIVE SCALAR IN 5D}

\section{A. The setup}

Following [5], we consider a massive scalar field in the 5-dimensional anti-de Sitter spacetime endowed with $\mathbf{Z}_{\mathbf{2}}$ symmetry across its boundary, described by the following interval

$$
d s^{2}=\Omega^{2}(z) \eta_{M N} d x^{M} d x^{N}, \quad \Omega(z)=\frac{L}{L+|z|},
$$

where the mostly minus metric convention is assumed. The coordinate $z$, parametrizing the fifth dimension ranges from $-\infty$ to $+\infty$ and the theory is constrained to be $\mathbf{Z}_{\mathbf{2}}$ invariant under the flip of sign $z \rightarrow-z$, under which the scalar is assumed to be even, $\phi(x, z)=\phi(x,-z)$. We are interested in the $4 \mathrm{D}$ effective theory on the physical brane, located at $z=0$, where $\phi$ is additionally coupled to a $4 \mathrm{D}$ source $j(x)$. The complete action thus reads

$$
\begin{aligned}
S_{\phi} & =\int d^{4} x \int_{-\infty}^{\infty} d z\left[\sqrt{-g}\left(\frac{1}{2} g^{M N} \partial_{M} \phi \partial_{N} \phi-\frac{1}{2} m_{5}^{2} \phi^{2}\right)+L \phi(x) j(x) \delta(z)\right] \\
& =\int d^{4} x \int_{-\infty}^{\infty} d z \Omega^{3}\left(\frac{1}{2} \partial_{\mu} \phi \partial_{\mu} \phi-\frac{1}{2}\left(\partial_{z} \phi\right)^{2}-\frac{1}{2} \Omega^{2} m_{5}^{2} \phi^{2}\right)+\int d^{4} x L \phi(x) j(x) .
\end{aligned}
$$

All 4D indices on the last line are assumed to be contracted with the flat 4D metric, and we do not distinguish between upper and lower $4 \mathrm{D}$ components. Varying this action with respect to $\phi$ yields the following equation of motion

$$
\left(-\square+\partial_{z}^{2}-\frac{3 \operatorname{sgn}(z)}{|z|+L} \partial_{z}-\frac{\left(m_{5} L\right)^{2}}{(|z|+L)^{2}}\right) \phi(x, z)=-L j(x) \delta(z)
$$

which, when integrated across the brane (that is, within the interval $z \in[-\epsilon, \epsilon]$, with $\epsilon \rightarrow 0$ ) implies the following boundary condition for $\phi$ :

$$
\left.\partial_{z} \phi\right|_{z=0}=-\frac{L}{2} j(x) .
$$

In order to derive the 4D effective action on the brane, we will need to solve the system (3) and (4). This is the subject of the next subsection.

\section{B. Kaluza-Klein modes}

Consistently with the equation of motion (3) and boundary condition (4), the 5D field $\phi$ can be decomposed in terms of the $4 \mathrm{D} \mathrm{KK}$ modes as follows:

$$
\phi(x, z)=\int_{0}^{\infty} d m L \phi^{(m)}(x) \chi_{\nu}^{(m)}(z),
$$

where we have defined

$$
\nu=\sqrt{4+\left(m_{5} L\right)^{2}} .
$$

The KK wave functions $\chi_{\nu}^{(m)}$ satisfy the following bulk equation

$$
\left(\partial_{z}^{2}-\frac{3 \operatorname{sgn}(z)}{|z|+L} \partial_{z}-\frac{\left(m_{5} L\right)^{2}}{(|z|+L)^{2}}\right) \chi_{\nu}^{(m)}(z)=-m^{2} \chi_{\nu}^{(m)}(z),
$$

complemented with the boundary condition at the brane

$$
\left.\partial_{z} \chi_{\nu}^{(m)}(z)\right|_{z=0}=0
$$

Explicitly, these KK wave functions read [5]

$$
\chi_{\nu}^{(m)}(z)=\sqrt{\frac{m L}{2}}\left(\frac{|z|+L}{L}\right)^{2}\left[a_{m} J_{\nu}(m(|z|+L))+b_{m} Y_{\nu}(m(|z|+L))\right],
$$

where the two coefficients $a_{m}$ and $b_{m}$ are given by the following expressions 


$$
\begin{aligned}
& a_{m}=-\frac{Y_{\nu-1}(m L)-\frac{\nu-2}{m L} Y_{\nu}(m L)}{\sqrt{\left(Y_{\nu-1}(m L)-\frac{\nu-2}{m L} Y_{\nu}(m L)\right)^{2}+\left(J_{\nu-1}(m L)-\frac{\nu-2}{m L} J_{\nu}(m L)\right)^{2}}} \\
& b_{m}=\frac{J_{\nu-1}(m L)-\frac{\nu-2}{m L} J_{\nu}(m L)}{\sqrt{\left(Y_{\nu-1}(m L)-\frac{\nu-2}{m L} Y_{\nu}(m L)\right)^{2}+\left(J_{\nu-1}(m L)-\frac{\nu-2}{m L} J_{\nu}(m L)\right)^{2}}} .
\end{aligned}
$$

Importantly, for nonzero bulk mass $m_{5}$ there is no normalizable zero mode, that is, no mode with $m=0$ [5]. (For $m_{5}$ strictly zero, such a mode does exist and would correspond to the $z$-independent bulk profile $\chi^{(0)}=$ const [4].) Nevertheless, even for a nonzero $m_{5}$, the spectrum features a gapless continuum of massive KK modes, whose wave functions satisfy the usual orthonormality conditions

$$
\int_{-\infty}^{\infty} d z\left(\frac{L}{|z|+L}\right)^{3} \chi_{\nu}^{(m)}(z) \chi_{\nu}^{\left(m^{\prime}\right)}(z)=\delta\left(m-m^{\prime}\right) \quad\left(m, m^{\prime}>0\right) .
$$

Plugging decomposition (5) into the original 5D action (2), one arrives at the 4D effective action for the KK continuum

$$
S_{\phi}=L \int d^{4} x \int_{0}^{\infty} d m L\left[\frac{1}{2}\left(\partial \phi^{(m)}\right)^{2}-\frac{m^{2}}{2}\left(\phi^{(m)}\right)^{2}+\chi^{(m)}(0) \phi^{(m)} j\right] .
$$

At each KK level, the canonically normalized 4D field $\phi^{(m)} / \sqrt{L}$ couples to the brane source $j$ with strength $\chi^{(m)}(0) / \sqrt{L}$. This coupling weakens for higher KK modes.

The force due to the exchange of massive KK modes between two four-dimensional brane sources is determined by the following amplitude (transformed to momentum space along the brane dimensions)

$$
\Delta_{\nu}\left(p^{2}\right)=\int_{0}^{\infty} d m L \frac{\left|\chi_{\nu}^{(m)}(0)\right|^{2}}{p^{2}-m^{2}+i \epsilon}=\frac{L^{2}}{2}\left[(p L) \frac{H_{\nu-1}^{(1)}(p L)}{H_{\nu}^{(1)}(p L)}-(\nu-2)\right]^{-1} \quad(\nu>2),
$$

where $p \equiv \sqrt{p^{2}}$. This formula, valid strictly for $\nu>2$, was first derived in Ref. [5]. Addressing the case of $\nu=2$, on the other hand, is subtle and will be our focus in the remainder of this section.

\section{Continuity in the bulk mass}

The main task of the present subsection is demonstrating continuity of the physical predictions of the theory in the $m_{5} \rightarrow 0$ limit. This limit is nontrivial: as we have remarked above, depending on whether the parameter $m_{5}$ is strictly zero or not, there is an extra zero mode present in the KK spectrum, leading to a discontinuity in the degrees of freedom in the 4D theory on the brane.

Reference [5] has demonstrated how exactly continuity occurs for nonzero, but small $m_{5}$ at the level of the Newtonian potential, experienced by brane sources due to the exchange of the scalar's KK modes. The crucial role is played by a special resonant mode, composed of the KK modes of the $4 \mathrm{D}$ theory. This resonance, which only exists for $m_{5} \neq 0$ (or $\nu \neq 2$ ), is characterized by mass and width, given by

$$
m_{0}^{2} \approx \frac{m_{5}^{2}}{2}, \quad \Gamma \approx \frac{\pi}{16 \sqrt{2} L}\left(m_{5} L\right)^{3}
$$

(Notice that for reasonable values of the bulk mass, $m_{5} \lesssim 1 / L$, the resonance's width is parametrically smaller than its mass.) The presence of the pole corresponding to the resonance (13) can be readily established by studying the analytic structure of the amplitude (12), see Ref. [5] for details. The negative imaginary part of the pole tends to zero as $m_{5} \rightarrow 0$, or equivalently as $\nu \rightarrow 2$ (of course, in this limit the real part vanishes as well and the pole asymptotically merges with the origin of the complex plane). Therefore, in the limit $\nu \rightarrow 2$ one can conveniently write Eq. (12) as

$\left.\Delta_{\nu}\left(p^{2}\right)\right|_{\nu \rightarrow 2}=\mathrm{P} . \mathrm{V} \cdot\left(\frac{L^{2}}{2} \frac{H_{2}^{(1)}(p L)}{(p L) H_{1}^{(1)}(p L)}\right)-i \pi \delta\left(p^{2}\right)$,

where P.V.(...) in the first term denotes the principal value of the expression in the parentheses, while the second term essentially provides the $i \epsilon$-prescription for the pole at $p^{2}=0$, corresponding to the $m_{5} \rightarrow 0$ limit of the resonance. To summarize, the analytic structure of the expression in (14) features the pole, corresponding to the $m_{5} \rightarrow 0$ limit of the 4D resonance scalar (responsible for the $r^{-1}$ piece in the static potential), as well as a branch cut, 


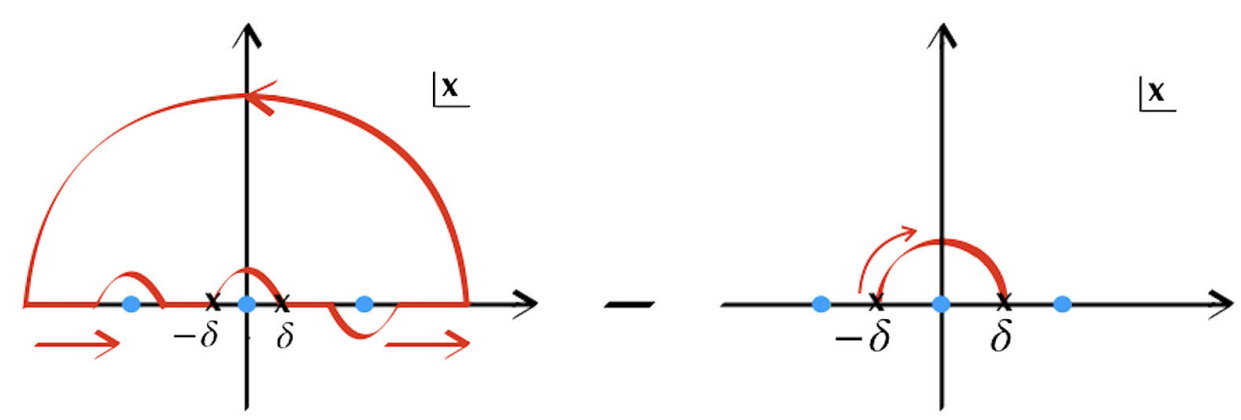

FIG. 1. A contour for computing the second term in Eq. (16).

corresponding to the entire gapless KK continuum (which leads to the $r^{-3}$ piece in the static potential).

We'd like to compare the above expression for the amplitude with its counterpart in the theory with strictly zero bulk scalar mass. To that end, one can study the KK spectrum just like we did for the massive bulk theory. As remarked above, in addition to the KK continuum there is now the true zero mode in the spectrum, and the amplitude becomes:

$$
\begin{aligned}
\Delta_{\nu=2}\left(p^{2}\right)= & \frac{1}{p^{2}+i \epsilon}+\int_{0}^{\infty} d m L \frac{\left|\chi_{2}^{(m)}(0)\right|^{2}}{p^{2}-m^{2}+i \epsilon} \\
= & \frac{1}{p^{2}+i \epsilon}+L^{2} \int_{0}^{\infty} d x \frac{1}{(p L)^{2}-x^{2}+i \epsilon} \\
& \times \frac{2}{\pi^{2} x\left(Y_{1}(x)^{2}+J_{1}(x)^{2}\right)},
\end{aligned}
$$

where the first term on the right-hand side of the first line comes from the $m=0$ zero mode exchange, while the second term-from the exchange of the $m>0 \mathrm{KK}$ modes. With the help of a few identities involving Bessel/Hankel functions, the second line of (15) can be massaged into an integral over (almost) the entire real axis

$$
\begin{aligned}
\Delta_{\nu=2}\left(p^{2}\right)= & \frac{1}{p^{2}+i \epsilon}+\frac{L^{2}}{2 \pi i} \lim _{\delta \rightarrow 0^{+}}\left(\int_{-\infty}^{-\delta} d x+\int_{\delta}^{+\infty} d x\right) \\
& \times \frac{1}{(p L)^{2}-x^{2}+i \epsilon} \frac{H_{2}^{(1)}(x)}{H_{1}^{(1)}(x)} .
\end{aligned}
$$

The integrand on the right-hand side of this expression has 3 poles, those at $x= \pm(p L+i \epsilon)$ and at $x=0$. The last of these poles is avoided by the $\delta$-prescription in the integral (16) (that is, by the fact that we are integrating up to $-\delta$, and from $+\delta$ ). The way we traverse the former two poles is determined by the standard Feynman ie prescription. Closing the integration contour by the large semicircle at infinity, as well as the small semicircle between $-\delta$ and $+\delta$, the integral can be readily computed. The integrand decays sufficiently fast at infinity so that the large semicircle contributes nothing. The small semicircle, on the other hand, does give a nonzero contribution, which has to be substracted to get the desired term in (16). This situation is schematically depicted in Fig. 1, where we chose to close the contour in the upper half of the complex plane-closing it in the lower half would lead to the same result, as can be straightforwardly checked. The contour on the left picks up the single pole at $x=p L+i \epsilon$. Computing the contribution of this pole and substracting from it the contribution of the semicircle on the right of Fig. 1, we finally get:

$$
\begin{aligned}
\Delta_{\nu=2}\left(p^{2}\right) & =\frac{1}{p^{2}+i \epsilon}+\frac{L^{2}}{2} \frac{H_{2}^{(1)}(p L)}{(p L) H_{1}^{(1)}(p L)}-\frac{1}{p^{2}} \\
& =\text { P.V. }\left(\frac{L^{2}}{2} \frac{H_{2}^{(1)}(p L)}{(p L) H_{1}^{(1)}(p L)}\right)-i \pi \delta\left(p^{2}\right),
\end{aligned}
$$

where the second and the third terms in the first line stem from the left and the right contours in Fig. 1 respectively, ${ }^{2}$ while the first term in the second line emerges from the three terms in the first line. Equation (17) gives the final form of the $m_{5}=0$ amplitude, which exactly agrees with the $m_{5} \rightarrow 0$ limit (14) of its counterpart in the theory with nonzero $m_{5}$. Since the considered scalar has no self interactions, and its interactions with the 5D graviton fluctuations are neglected, the above-established continuity of a two-point amplitude establishes continuity of the theory in the bulk scalar mass.

\section{The static potential}

It is instructive to understand continuity of the theory in the limit $m_{5} \rightarrow 0$ on a simple physical observable- the static Newtonian potential between brane sources. We will start with looking at the large-distance limit of the amplitude (12), corresponding to $p L \ll 1$. Furthermore, we will also assume that the bulk mass is small compared to AdS curvature, $\nu-2 \ll 1$. One can then expand $\Delta_{\nu}\left(p^{2}\right)$ as follows

\footnotetext{
${ }^{2}$ It is straightforward to see that the semicircle on the right panel of Fig. 1 provides a finite contribution to the integral. To that end, note that $H_{2}^{(1)} / H_{1}^{(1)} \propto x^{-1}$ in the limit $x \rightarrow 0$, and the integral over the small semicircle $s_{1}$ is proportional to $\int_{s_{1}} d x / x=-i \pi$.
} 


$$
\Delta_{\nu}\left(p^{2}\right) \approx \frac{L^{2}}{(p L)^{2}(1-(\nu-2))-2(\nu-2)-\frac{(p L)^{4}}{2} \ln (p L / 2 i)},
$$

where the corrections to the denominator are of order $(p L)^{6},(\nu-2)^{2}(p L)^{2}$ and $(\nu-2)(p L)^{4}$. Keeping only terms of order $p L$ and $\nu-2$ in (18), this gives rise to the Yukawa potential

$$
V_{1}(r)=\int \frac{d^{3} \mathbf{p}}{(2 \pi)^{3}} e^{i \overrightarrow{\mathbf{p}} \cdot \vec{r}} \frac{L^{2}}{-(\mathbf{p} L)^{2}-2(\nu-2)} \approx-\frac{e^{-m_{0} r}}{4 \pi r}
$$

In general, the correction $V_{2}$ to this expression depends on the relative magnitude of the two expansion parameters. In what follows, we will focus on deriving two different limits of this correction. ${ }^{3}$

\section{Large distances: $r \gg m_{5}^{-1}$}

At large distances, momentum transfer is the smallest scale in the problem, in particular $p L \ll \nu-2$, and expanding the amplitude (18) in momentum yields:

$$
\begin{aligned}
V\left(r \gg m_{5}^{-1}\right) & =V_{1}+\int \frac{d^{3} \mathbf{p}}{(2 \pi)^{3}} e^{i \mathbf{p} \cdot \mathbf{r}} \frac{L^{2}(\mathbf{p} L)^{4} \ln (|\mathbf{p}| L / 2)}{8(\nu-2)^{2}} \\
& =V_{1}-\frac{45 L^{5}}{4 \pi(\nu-2)^{2} r^{7}} .
\end{aligned}
$$

At distances under consideration, $r \gg m_{5}^{-1}$, the Yukawa potential $V_{1}$ is contributed mainly by modes with $m \gtrsim m_{5}$ and is exponentially suppressed. The leading contribution to the potential is thus given by the second term in (20), as discussed in Ref. [5].

\section{Small bulk mass: $m_{\mathbf{5}} \rightarrow \mathbf{0}$}

The previous, large distance limit $r \gg m_{5}^{-1}$ is obviously not consistent with the massless limit of the bulk scalar. This limit, not considered in [5], is our main focus in the present section. When $m_{5}$ is sent to zero (that is, when $m_{5}$ is the smallest scale in the problem, but not strictly zero), both the mass and the width of the 4D resonance tend to zero as well, the latter vanishing faster than the former. The Yukawa potential of Eq. (19) therefore turns into the Newtonian one:

$$
\left.V_{1}(r)\right|_{m_{5} \rightarrow 0}=-\frac{1}{4 \pi r} .
$$

While this visually resembles the potential due to the exchange of a 4D massless scalar, it is important to stress

\footnotetext{
${ }^{3}$ It is important to note that the term "correction" for the extra term will not be adequate at distances $r \gg m_{0}$ at which the Yukawa potential is exponentially suppressed. In such a regime, the extra term $V_{2} \propto r^{-7}$ provides the leading contribution, as stressed in the discussion to follow.
}

that in the setup under consideration there is no localized massless mode in the problem. The bulk mass $m_{5}$ is far smaller than any other scale, but it is still not strictly zerothe KK spectrum therefore does not possess a massless state (zero mode), and the potential (21) is due to a linear combination of KK modes with $m>0$. The correction to the Newtonian potential $V_{1}$ can be found by expanding the $\nu \rightarrow 2$ limit of the amplitude (18)

$$
V_{2}(r)=\frac{L^{2}}{2} \int \frac{d^{3} \mathbf{p}}{(2 \pi)^{3}} e^{i \mathbf{p} \cdot \mathbf{r}} \ln (|\mathbf{p}| L / 2)=-\frac{L^{2}}{8 \pi r^{3}} .
$$

Summing up the two limiting contributions in (21) and (22) yields the expression, equivalent to what one would get for the static potential in the case of a strictly massless bulk scalar. As we have remarked above, in the latter case there does exist a zero mode in the four-dimensional spectrum, and it leads to the Newtonian force, equivalent to (21). Moreover, in the $m_{5}=0$ theory, the gapless continuum of KK modes works exactly as it does in the case of a small but nonzero $m_{5}$, providing a correction, equivalent to (22). We have therefore established continuity between the two theories with strictly vanishing $m_{5}$ and however small, but nonzero $m_{5}$ : the physical spectra of these theories are somewhat different, but all observables - in particular the gravitational potential between brane sources-are perfectly continuous.

\section{MASSIVE SCALAR WITH A ZERO MODE}

Formally, even for nonvanishing bulk mass $m_{5}$, the bulk equation (6) for the scalar's 4D KK modes admits a wouldbe (normalizable) zero mode solution with the $z$-profile proportional to $(|z|+L)^{-\nu+2}$. As already remarked in Sec. II, however, this solution is incompatible with the boundary condition (7), which effectively removes it from the physical KK spectrum. This observation suggests that the entire theory may be made compatible with the existence of the zero mode-even for nonzero bulk mass -by modifying the boundary part of the original scalar action (a similar mechanism has been considered for the case of a massive bulk vector in [6]).

In what follows, we will show that modifying the theory by an extra scalar mass term, localized at $z=0$ does the job of reintroducing the zero mode on the brane. Indeed, consider the following theory

$$
S=S_{\phi}+\frac{\nu-2}{2 L} \int_{z=0} d^{4} x \phi^{2},
$$

where $S_{\phi}$ denotes the action of the "minimal" massive theory (2). Notice also, that the new boundary term has a "tachyonic" sign, which, however, does not lead to inconsistency of the theory-indeed, we will show that the effects of the "wrong" sign boundary mass will be overwhelmed by the effects of the "correct" sign bulk mass in $S_{\phi}$. Physically, the tachyonic mass can be thought of as giving an additional attractive contribution to the effective 
("volcano") potential that traps the scalar zero mode on the brane.

With the additional boundary term, the dynamics is governed by the modified equation of motion

$$
\begin{aligned}
& \left(-\square+\partial_{z}^{2}-\frac{3 \operatorname{sgn}(z)}{|z|+L} \partial_{z}-\frac{\left(m_{5} L\right)^{2}}{(|z|+L)^{2}}+\frac{\nu-2}{L} \delta(z)\right) \phi(x, z) \\
& =-L j(x) \delta(z),
\end{aligned}
$$

which yields the following boundary condition on the brane

$$
\left.\left(\partial_{z}+\frac{\nu-2}{L}\right) \phi\right|_{z=0}=-\frac{L}{2} j(x)
$$

The KK mode wave functions can be found by solving the system (24) and (25) in the absence of sources, which establishes that the 4D spectrum of the theory indeed consists of the zero mode scalar, in addition to the continuum of massive KK states. The bulk profiles for these modes read

$$
\begin{aligned}
\chi_{\nu}^{(0)}(z) & =\sqrt{\nu-1}\left(\frac{L}{|z|+L}\right)^{\nu-2}, \\
\chi_{\nu}^{(m)}(z) & =\sqrt{\frac{m L}{2}}\left(\frac{|z|+L}{L}\right)^{2}\left[\frac{J_{\nu-1}(m L) Y_{\nu}(m(|z|+L))-Y_{\nu-1}(m L) J_{\nu}(m(|z|+L))}{Y_{\nu-1}(m L)^{2}+J_{\nu-1}(m L)^{2}}\right],
\end{aligned}
$$

where, to avoid notational clutter, we have kept the same notation for the KK wave functions as in the previous section, although the corresponding functions are of course different in the modified theory at hand. Plugging the KK decomposition $\phi(x, z)=\phi^{(0)} \chi_{\nu}^{(0)}(z)+\int_{0}^{\infty} d m L \phi^{(m)}(x) \chi_{\nu}^{(m)}(z)$ into the original action (23) and using the standard orthonormality properties of the KK wave functions, we arrive at the $4 \mathrm{D}$ effective theory of the following form

$$
\begin{aligned}
S= & L \int d^{4} x\left[\frac{1}{2}\left(\partial \phi^{(0)}\right)^{2}+\sqrt{\nu-1} \phi^{(0)} j\right. \\
& \left.+\int_{0}^{\infty} d m L\left(\frac{1}{2}\left(\partial \phi^{(m)}\right)^{2}-\frac{m^{2}}{2}\left(\phi^{(m)}\right)^{2}+\chi_{\nu}^{(m)}(0) \phi^{(m)} j .\right)\right],
\end{aligned}
$$

One can see, that all modes are well-behaved despite the "wrong" sign of the brane mass term in (23). Moreover, the canonically normalized zero mode and the massive KK modes couple to brane sources with strength, set by the quantities $\sqrt{(\nu-1) / L}$ and $\chi^{(m)}(0) / \sqrt{L}$ respectively. Exchange of these modes between brane sources gives rise to the following amplitude

$$
\begin{aligned}
\Delta\left(p^{2}\right) & =\frac{\nu-1}{p^{2}+i \epsilon}+\int_{0}^{\infty} d m L \frac{\left|\chi^{(m)}(0)\right|^{2}}{p^{2}-m^{2}+i \epsilon} \\
& =\frac{\nu-1}{p^{2}+i \epsilon}+\frac{2 L^{2}}{\pi^{2}} \int_{0}^{\infty} d x \frac{1}{(p L)^{2}-x^{2}+i \epsilon} \frac{1}{x\left(Y_{\nu-1}(x)^{2}+J_{\nu-1}(x)^{2}\right)} .
\end{aligned}
$$

By performing manipulations on the second term, very similar to the ones discussed in the previous section, one can integrate over $x$ thereby arriving at the final, closed-form expression for the amplitude

$$
\Delta\left(p^{2}\right)=\mathrm{P} . \mathrm{V} \cdot\left(\frac{L^{2}}{2} \frac{H_{\nu}^{(1)}(p L)}{(p L) H_{\nu-1}^{(1)}(p L)}\right)-i \pi(\nu-1) \delta\left(p^{2}\right) .
$$

This expression differs by the order of the Hankel functions involved, as well as by the strength of the massless pole, from its counterpart (17) in the massless bulk theory, described in the previous section.

This setup is fundamentally different from the previous setup in that the number of degrees of freedom is continuous in the massless limit. There is a proper zero mode all along and therefore, no puzzle to resolve regarding the question of why the physical amplitudes are continuous. However, we include a discussion of this setup for a couple of reasons. The first is that when looked at from the perspective of the analogue Schrodinger potential as was originally done for the RS scenario [1], it is interesting to see how a bound state arises at the top of the "volcano" even for nonzero bulk mass. Second, we will find that in the follow up to this paper when we consider the warped 
geometry scenario for higher spin fields, this toy model will be quite helpful in understanding the tensor case.

\section{DE SITTER BRANE AND COSMOLOGY}

The above discussion has exclusively concerned the case of a flat brane, which requires tuning the brane tension $\lambda$ against the bulk cosmological constant $\Lambda$. It is known due to Kaloper [7] and Nihei [8], that upon detuning these two quantities, one can end up with an inflating (de Sitter) brane, instead of a flat one. The metric of the corresponding spacetime reads $[7,8]$ :

$$
\begin{aligned}
d s^{2}= & \left(\cosh \frac{y}{L}-q \sinh \frac{y}{L}\right)^{2}\left(-d t^{2}+e^{2 H t} d \vec{x}^{2}\right)+d y^{2}, \\
& \left(q \equiv \sqrt{1+(H L)^{2}}\right),
\end{aligned}
$$

where the brane is located at $y=0$ in the given coordinates, $L$ is the curvature radius of the bulk and $H$ is the (constant) Hubble rate on the $d S_{4}$ spacetime on the brane world volume, determined by $\Lambda$ and $\lambda$-the precise relation will not be important for our purposes.

It will prove convenient to perform a change of coordinates

$$
e^{y / L}=\frac{q+1}{H L} \frac{e^{H\left(z+z_{0}\right)}-1}{e^{H\left(z+z_{0}\right)}+1}, \quad z_{0} \equiv H^{-1} \ln \frac{q+H L+1}{q-H L+1},
$$

which removes the coordinate singularity in the line element (30), putting it into the following form

$$
d s^{2}=\frac{(H L)^{2}}{\sinh ^{2} H\left(z+z_{0}\right)}\left(-d t^{2}+e^{2 H t} d \vec{x}^{2}+d z^{2}\right) .
$$

In the new coordinates, the brane is located at $z=0$, and we will assume that the theory is invariant under reflections of this coordinate, $z \rightarrow-z$, so (32) should be viewed as describing the brane's "positive side," which we will exclusively work with in the following discussion. On this side of the brane, the $z$ coordinate ranges from 0 to $\infty$ as $y$ ranges from zero to a finite value, determined by the location of the coordinate singularity in (30), the Rindler horizon. Hence, the $z$ coordinate does not cover the entire space covered by the $y$ coordinate, but only its patch ranging from the origin to the Rindler horizon.
While we could continue the spacetime past the horizon to values of $y>L \ln \left(\frac{q+1}{H L}\right)$, anything happening beyond this region would not effect the observers on the brane. While a signal sent from the brane to the horizon would take a finite proper time to arrive, it would take an infinite amount of time according to an observer on the brane. Furthermore, the boundary conditions for the KK modes are already completely determined at the horizon by the condition of normalizability so we set aside the question of any extension of this spacetime beyond the horizon as irrelevant for our purposes.

Consider the Euler-Lagrange equation of motion for a massive, $z$-reflection-even bulk scalar $\phi(x, z)=\sigma(x) \chi(z)$ in the background (32):

$$
\frac{d^{2} \chi}{d u^{2}}-3 \frac{\cosh u}{\sinh u} \frac{d \chi}{d u}+\left(\frac{m^{2}}{H^{2}}-\frac{m_{5}^{2} L^{2}}{\sinh ^{2} u}\right)=0,
$$

where, with the Kaluza-Klein decomposition in mind, we have defined $\square_{4} \sigma=m^{2} \sigma$, as well as $\bar{z} \equiv z+z_{0}$ and $u \equiv H \bar{z}$. To further simplify notation, we will also define

$$
A^{2}=\frac{m^{2}}{H^{2}}, \quad B^{2}=m_{5}^{2} L^{2},
$$

so that the equation of interest (33) becomes

$$
\chi^{\prime \prime}-3 \frac{\cosh (u)}{\sinh (u)} \chi^{\prime}+\left(A^{2}-\frac{B^{2}}{\sinh ^{2}(u)}\right) \chi=0, \quad \chi^{\prime} \mid=0 .
$$

Here, the last equation - the boundary condition on the brane-can be obtained by integrating the bulk equation across the brane and keeping in mind that $\chi$ is a $z$-reflection-even field (again, the vertical stroke denotes evaluation at $\left.u=H z_{0}\right)$. A solution to this equation with given $m^{2}$ (that is, given value of the parameter $A^{2}$ ) describes a localized, normalizable mode if it satisfies

$$
\int_{0}^{\infty} d z \sqrt{g} g^{00} \chi^{2}=\text { finite }
$$

This imposes a second boundary condition, this time at $u \rightarrow \infty$, on normalizable modes. In what follows, we will be looking for precisely such localized modes.

A general solution to (35) reads

$$
\begin{aligned}
\chi(u)= & \cosh (u)^{\frac{1}{2}\left(3-\sqrt{9-4 A^{2}}\right)}(\tanh u)^{2-\sqrt{4+B^{2}}} \\
& \times\left[C_{2} \cdot{ }_{2} F_{1}\left(a_{1}, b_{1}, c_{1}, t\right)-C_{1} \cdot(\tanh u)^{2 \sqrt{4+B^{2}}} \cdot{ }_{2} F_{1}\left(a_{2}, b_{2}, c_{2}, t\right)\right],
\end{aligned}
$$

where $C_{1}$ and $C_{2}$ are the two integration constants and the coefficients $a_{1,2}, b_{1,2}$, and $c_{1,2}$, together with the $u$-dependent quantity $t$ have been defined as follows 


$$
\begin{array}{lll}
a_{1}=\frac{1}{4}\left(1+\sqrt{9-4 A^{2}}-2 \sqrt{4+B^{2}}\right), & b_{1}=\frac{1}{4}\left(3+\sqrt{9-4 A^{2}}-2 \sqrt{4+B^{2}}\right), & c_{1}=1-\sqrt{4+B^{2}}, \\
a_{2}=\frac{1}{4}\left(1+\sqrt{9-4 A^{2}}+2 \sqrt{4+B^{2}}\right), & b_{2}=\frac{1}{4}\left(3+\sqrt{9-4 A^{2}}+2 \sqrt{4+B^{2}}\right), & c_{2}=1+\sqrt{4+B^{2}},
\end{array}
$$

and $t=\tanh ^{2} u$. In order to fix the integration constants $C_{1}$ and $C_{2}$, we will need to study the behavior of the solution (37) for both large and small values of $u$-something we will turn to next.

\section{A. Large $u$}

For large $u$ (corresponding to $t=\tanh ^{2} u \rightarrow 1^{-}$), and for $c-a-b<0$ which is the case for the solution (37), ${ }^{4}$ the relevant limit of the hypergeometric function reads

$\lim _{t \rightarrow 1^{-2}} F_{1}(a, b, c, t)=(1-t)^{c-a-b} \frac{\Gamma(c) \Gamma(a+b-c)}{\Gamma(a) \Gamma(b)}$,

which gives in our case:

$$
\begin{aligned}
& \lim _{u \rightarrow \infty} F_{1}\left(a_{1,2}, b_{1,2}, c_{1,2}, \tanh ^{2}(u)\right) \\
& =(\cosh u)^{\sqrt{9-4 A^{2}}} \frac{\Gamma\left(c_{1,2}\right) \Gamma\left(\sqrt{9-4 A^{2}} / 2\right)}{\Gamma\left(a_{1,2}\right) \Gamma\left(b_{1,2}\right)} .
\end{aligned}
$$

Plugging this expression into the general solution then yields

$$
\begin{aligned}
\chi(u \gg 1) \simeq & \Gamma\left(\sqrt{9-4 A^{2}} / 2\right)(\cosh u)^{\frac{1}{2}\left(3+\sqrt{9-4 A^{2}}\right)} \\
& \times\left(C_{2} \frac{\Gamma\left(c_{1}\right)}{\Gamma\left(a_{1}\right) \Gamma\left(b_{1}\right)}-C_{1} \frac{\Gamma\left(c_{2}\right)}{\Gamma\left(a_{2}\right) \Gamma\left(b_{2}\right)}\right) .
\end{aligned}
$$

This correctly reproduces one of the growing modes at large $u$. To see this, we note that when $u \rightarrow \infty$, the two independent solutions have the form $\exp \left(k_{1,2} x\right)$, where $k_{1}$ and $k_{2}$ are the two solutions of the quadratic equation $k^{2}-3 k x+A^{2}=0$; explicitly, $k_{1,2}=\left(3 \pm \sqrt{9-4 A^{2}}\right) / 2$ and one can see that (40) reproduces the $k_{1}$-mode, while the $k_{2}$-mode corresponds to the other, subleading solution. The latter solution describes a localized, normalizable mode [with our proper definition of normalizability, given in Eq. (36)], while the former corresponds to a nonnormalizable mode and should thus be removed. To that end, we need to tune $C_{1}$ and $C_{2}$ as follows:

$$
C_{2}=C_{1} \frac{\Gamma\left(a_{1}\right)}{\Gamma\left(a_{2}\right)} \frac{\Gamma\left(b_{1}\right)}{\Gamma\left(b_{2}\right)} \frac{\Gamma\left(c_{2}\right)}{\Gamma\left(c_{1}\right)} .
$$

With this tuning at hand, one can go ahead and study the behavior of the solution in the opposite limit-the one corresponding to $u \ll 1$.

\footnotetext{
${ }^{4}$ Note that $c_{1}-a_{1}-b_{1}=c_{2}-a_{2}-b_{2}=-\sqrt{9-4 A^{2}} / 2$.
}

\section{B. Small $u$}

Let us now try to understand whether one can satisfy the boundary condition in Eq. (35)

$$
\chi^{\prime} \mid \equiv \chi^{\prime}(\epsilon)=0 .
$$

Note, importantly, that the value of $u$ on the brane is small

$$
\epsilon \equiv H z_{0} \sim H L \ll 1,
$$

and let us first try to understand the solution analytically. For $x \ll 1$ our equation becomes

$$
\chi^{\prime \prime}-\frac{3}{u} \chi^{\prime}-\frac{B^{2}}{u^{2}} \chi=0
$$

which is solved by

$$
\chi(u \ll 1) \simeq \tilde{C}_{1} u^{2-\sqrt{4+B^{2}}}+\tilde{C}_{2} u^{2+\sqrt{4+B^{2}}},
$$

where $\tilde{C}_{1,2}$ will be expressed in terms of $C_{1,2}$ for our particular solution of interest-we will give the explicit expressions for these coefficients below. Importantly, neither of the $\tilde{C}_{1,2}$ automatically vanish for our solution, and the (derivatives of the) two terms can balance each other to satisfy the boundary condition (42) at $u=\epsilon$. Taylor-expanding the solution (37) and using (41), we have

$$
\begin{aligned}
& C_{1}^{-1} \chi^{\prime}(\epsilon) \\
& =\left(2-\sqrt{4+B^{2}}\right) \frac{\Gamma\left(a_{1}\right) \Gamma\left(b_{1}\right) \Gamma\left(c_{2}\right)}{\Gamma\left(a_{2}\right) \Gamma\left(b_{2}\right) \Gamma\left(c_{1}\right)} \epsilon^{-\sqrt{4+B^{2}}}\left(\epsilon+\mathcal{O}\left(\epsilon^{3}\right)\right) \\
& -\left(2+\sqrt{4+B^{2}}\right) \epsilon^{\sqrt{4+B^{2}}}\left(\epsilon+\mathcal{O}\left(\epsilon^{3}\right)\right) .
\end{aligned}
$$

(The precise expression for $C_{1}$-not important for the discussion to come-can be found from orthonormality of the AdS mode functions.) Examining this expression, one can see that there certainly exist possibilities for it to vanish, one of which can be understood as follows: the two terms balance each other for

$$
\epsilon^{2 \sqrt{4+B^{2}}}=\frac{2-\sqrt{4+B^{2}}}{2+\sqrt{4+B^{2}}} \frac{\Gamma\left(a_{1}\right) \Gamma\left(b_{1}\right) \Gamma\left(c_{2}\right)}{\Gamma\left(a_{2}\right) \Gamma\left(b_{2}\right) \Gamma\left(c_{1}\right)} ;
$$

under our assumptions, $\epsilon$ is small, and so should be the right-hand side of this expression. There seems to be at least the following possibility for this: $2-\sqrt{4+B^{2}}$ is small if $B$ is small and on top of that $\Gamma\left(c_{1}\right)$ is large, which 
makes the left-hand side small for a generic choice of $A$. Of course, for this to be true, one has to check that there are no surprises at higher orders in Taylor expansion in $\epsilon$. We can prove the absence of such surprises numerically by examining how the exact (unexpanded) $C_{1}^{-1} \chi^{\prime}(x)$ behaves for different $A$ and $B$.

\section{C. (Numerical) study of the exact expression for $\boldsymbol{y}^{\prime}(\boldsymbol{x})$}

The closed-form expression for $C_{1}^{-1} \chi^{\prime}(x)$ is quite cumbersome and we will not reproduce it here. Instead, let us choose a representative value for $\epsilon$, such as $\epsilon=10^{-4}$, and numerically explore the dependence of this expression on $A$ and $B$.

This dependence is shown, in the form of a 3D plot, on Fig. 2 (where irregularities arise whenever $a_{1}$ or $b_{1}$ cross negative integers). One can see that the function of interest comes close to zero for small $\mathrm{B}$, but it is not clear enough whether it actually crosses zero. To see whether $C_{1}^{-1} \chi^{\prime}(x)$ crosses zero, let us choose a particular value $B=10^{-4}$ and see if a value for $A$ exists such that this function vanishes.

The dependence of $C_{1}^{-1} \chi^{\prime}(x)$ on $A$ for $\epsilon=B=10^{-4}$ is shown on Fig. 3. It is clear that a value of $A$ exists, such that the desired function smoothly crosses zero-exactly like we predicted from the Taylor expansion argument, given above. (We emphasize again that the present argument is not using any approximation-we are working with exact expressions here.)

This establishes the presence of a localized mode for the case of a de Sitter brane. It is straightforward to see that the continuum of the KK excitations in this case starts above a gap determined by the $4 \mathrm{D}$ curvature scale:

$$
m_{K K}^{2} \geq \frac{9 H^{2}}{4},
$$

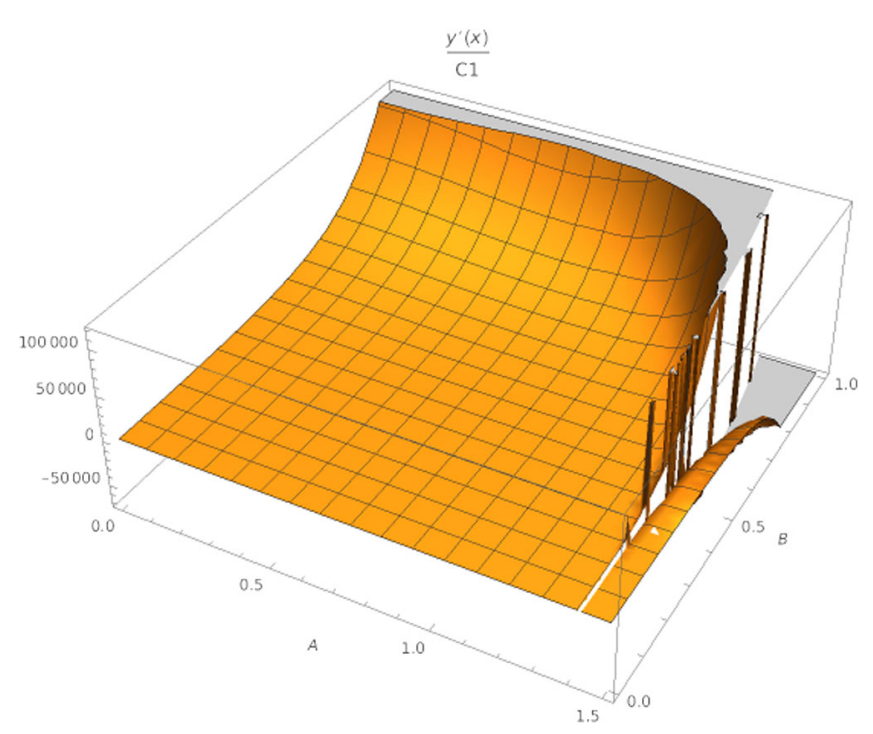

FIG. 2. The dependence of $C_{1}^{-1} y^{\prime}(x)$ on the parameter $A$ for $B=\epsilon=10^{-4}$.

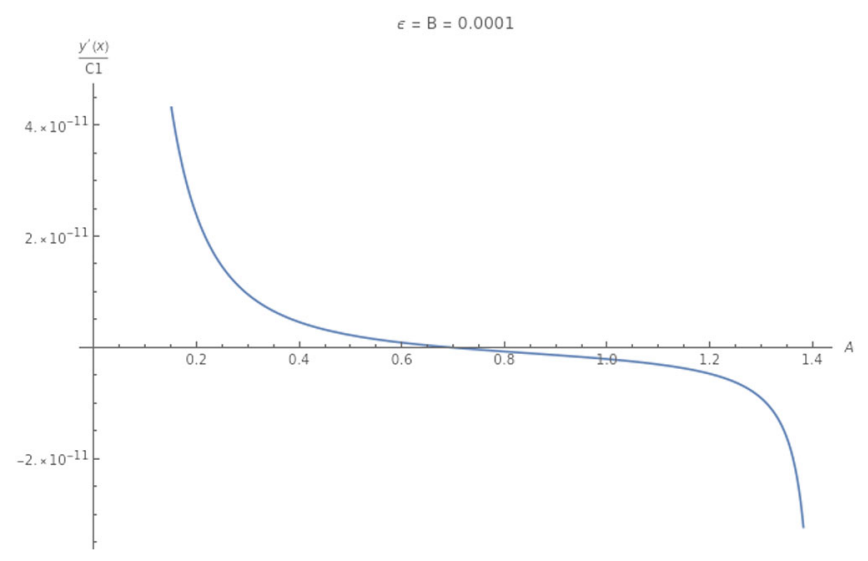

FIG. 3. The dependence of $C_{1}^{-1} y^{\prime}(x)$ on the parameters $A$ and $B$.

and the localized mode resides within the gap. It is difficult to calculate the mass of the localized mode. However, one can deduce scaling dependence of the mass of this mode on other parameters by approximately matching asymptotic solutions. Doing so, one obtains for the mass of the localized mode, $m_{*} \sim m /(H L)$, in the approximation when $H L \ll 1$ and $m_{*} \ll 3 H / 2$. Thus, in the above regime of the parameter space the mass of the localized mode is significantly larger than the mass of the bulk mode, $m$, but is smaller than the curvature scale $H$.

\section{Comments on cosmology}

We will outline here how the $5 \mathrm{D}$ massive scalar in $\operatorname{AdS}_{5} / Z_{2}$ can be used to describe an accelerated expansion of the universe. The rate of the acceleration will depend on the scalar mass, and therefore, choosing the value of the mass one can either make the expansion suitable for inflation, or for the late time acceleration.

To begin with consider the 5D massive scalar embedded in the geometry with a tuned brane and bulk, as in Sec. II, so that the 4D brane world-volume spacetime is flat. It remains flat as long as the scalar is in its vacuum state, $\phi=0$.

Let us now imagine that at some earlier time the initial value of the scalar was nonzero, $\phi=\phi_{0}$, with its time derivative being negligible. At that initial moment the scalar would add to the bulk energy density a positive quantity

$$
\Delta E=\frac{1}{2} m_{5}^{2} \phi_{0}^{2}
$$

For simplicity, we will assume that this energy density is less in its magnitude than the bulk AdS negative energy density; thus, the quadratic scalar potential will lead to a reduction of the magnitude of the negative energy density in the bulk. As a result, the bulk energy density and the brane tension will no longer be tuned, and the brane world volume will at that time moment acquire a positive spacetime curvature $[7,8]$ 


$$
H=\sqrt{\frac{\Delta E}{M_{5}^{3}}},
$$

where $M_{5}$ denotes the (specifically normalized) Planck mass of the 5D theory. Let us furthermore assume that after the initial moment the 5D scalar field is going to roll down its quadratic potential slowly during some reasonable classical interval of time. As a result of the slow, roll the curvature of the 5D spacetime will change slowly too, and so will the curvature of the 4D (quasi) de Sitter universe. This expansion can potentially describe either inflation in the early universe, or the dark energy driven acceleration, depending on the values of the scalar mass and $\phi_{0}$.

While we will not pursue the detailed studies of these cosmologies here, we point out two peculiarities of the proposed scheme: first the 4D Planck constant will also be changing in time as the 5D scalar rolls down its slope. Second, the 4D fluctuations, as shown in Sec. IV, will consist of one localized massive mode below the gap and the KK continuum above a gap. If applied to inflation, The localized mode will then be responsible for density perturbations; its mass is parametrically different from the mass of the 5D field that is rolling down. Thus, it might be interesting to work out the details of such a cosmological model.

\section{ACKNOWLEDGMENTS}

We thank Nemanja Kaloper for useful correspondence. G. G. is supported by the NSF Grant No. PHY-1915219. The work of D. P. and D. O. at N. Y. U. was supported by the Simons Foundation under the "Origins of The Universe" program.
[1] L. Randall and R. Sundrum, A Large Mass Hierarchy from a Small Extra Dimension, Phys. Rev. Lett. 83, 3370 (1999).

[2] S. J. Avis, C. J. Isham, and D. Storey, Quantum field theory in anti-de Sitter space-time, Phys. Rev. D 18, 3565 (1978).

[3] K. Behrndt and M. Cvetic, Supersymmetric domain wall world from D $=5$ simple gauged supergravity, Phys. Lett. B 475, 253 (2000).

[4] B. Bajc and G. Gabadadze, Localization of matter and cosmological constant on a brane in anti-de Sitter space, Phys. Lett. B 474, 282 (2000).
[5] S. L. Dubovsky, V. A. Rubakov, and P. G. Tinyakov, Brane world: Disappearing massive matter, Phys. Rev. D 62, 105011 (2000).

[6] B. Batell and T. Gherghetta, Localized U(1) gauge fields, millicharged particles, and holography, Phys. Rev. D 73, 045016 (2006).

[7] N. Kaloper, Bent domain walls as brane worlds, Phys. Rev. D 60, 123506 (1999).

[8] T. Nihei, Inflation in the five-dimensional universe with an orbifold extra dimension, Phys. Lett. B 465, 81 (1999). 